\title{
DIE DEUTSCH-DEUTSCHEN HISTORIKERBEZIEHUNGEN ZWISCHEN ABSCHLIESSUNG UND ÖFFNUNG
}

Anders als nach dem Ersten Weltkrieg erlebte die deutsche Geschichtswissenschaft nach dem Zweiten Weltkrieg keine anhaltende Isolierung, sondern im Gegenteil die denkbar rasche Wiederaufnahme in die communauté scientifique. Der Preis dieser Reintegration auf internationaler Ebene lag allerdings in der Desintegration auf nationaler Ebene, und die beiden so gegenläufigen Entwicklungen lassen sich gleichermaßen auf den Kalten Krieg und die Verfestigung zweier gegensätzlicher gesellschaftlicher Hemisphären zurückführen. Schon aus diesem Grund läßt sich mit dem von Karl Dietrich Erdmann geprägten Begriff der »Ökumene der Historiker « ${ }^{1}$ die disziplinäre Entwicklung zwischen 1945 und 1989/90 nicht vollständig begreifen. Der Terminus bildet eher eine geschichtspolitische Hoffnung in der Zeit der Teilung ab als eine analytische Kategorie der Forschung über sie. Mit welchen Begriffen und Konzepten aber kann das Miteinander und Gegeneinander zweier vielfach aufeinander bezogener und ineinander verwobener Fachentwicklungen westlich und östlich der politischen Demarkationslinie in der zweiten Hälfte des 20. Jahrhunderts erfaßt werden?

Den differenziertesten Ansatz bietet bis heute der von Christoph Kleßmann formulierte Vorschlag, die doppelte deutsche Geschichte in der Zeit ihrer Teilung als asymmetrisch verflochtene Parallel- und Kontrastgeschichte zu schreiben ${ }^{2}$. Allerdings ist er nur auf die bereits geschiedenen Geschichtswissenschaften anwendbar und kann deren Weg in die Teilung selbst nicht aufnehmen. Doch der Weg von der Einheit in die Doppelung der deutschen Geschichtswissenschaft nach 1945 ist lang, und er wirft die Frage auf, von welchem Zeitpunkt an die disziplinäre Spaltung so weit vorangeschritten war, $\mathrm{da} ß$ von zwei deutschen Historiographien gesprochen werden kann. Eine Antwort ist nicht ohne Differenzierung möglich, denn die inhaltliche Teilung der deutschen Geschichtswissenschaft in eine gezähmt-historistische, also »bür-

1 Vgl. Karl Dietrich ERDMANN, Die Ökumene der Historiker. Geschichte der Internationalen Historikerkongresse und des Comité international des sciences historiques, Göttingen 1987.

2 Vgl. Christoph KLessmanN, Verflechtung und Abgrenzung. Aspekte der geteilten und zusammengehörigen deutschen Nachkriegsgeschichte, in: Aus Politik und Zeitgeschichte B 29-30/1993, S. 30-41. 
gerliche« Richtung im Westen und eine marxistisch-leninistische, also »sozialistische « Richtung im Osten, ging der institutionellen Spaltung des Faches voraus. Beide Separierungen wurden zudem relativiert durch einzelne, noch lange und teils bis 1989 fortbestehende Gemeinschaftsunternehmen auf dem Gebiet der Bibliographie, der Quellenerschließung oder der Regionalgeschichte und auch der Volkskunde.

I.

Während die westliche Geschichtswissenschaft nach der Überwindung eines sogenannten Katastrophendenkens, das der östlichen Miseretheorie entsprach, institutionell und fachlich an die Weimarer Zeit anknüpfte, wurde die ostdeutsche Disziplin in verschiedenen Entwicklungsstufen radikal umgestaltet. In den ersten drei Jahren nach dem Zusammenbruch der NS-Herrschaft dominierten politische Reinigungsbemühungen, die sich durch geringe Kohärenz auszeichneten und mit der Betonung humanistisch-demokratischer Leitbilder Marxisten und Nichtmarxisten gleichermaßen zu umfassen suchten. Als das Fach Geschichte zum Wintersemester 1946/47 auf den von der Sowjetischen Militäradministration (SMAD) kontrollierten Hochschulen der Sowjetischen Besatzungszone (SBZ) wieder zugelassen wurde, hatte sich die Zusammensetzung von Studentenschaft und Lehrkörper bereits deutlich zugunsten von SED-Mitgliedern verändert. Ihren eigenen, parteikommunistischen Nachwuchs bildete die SED in dieser Zeit trotzdem weitgehend außerhalb der Hochschulen aus, da dort ihr Einfluß immer noch vergleichsweise gering war. Bis 1950 läßt sich in Bezug auf die ostdeutsche Geschichtswissenschaft von einer Sattelzeit sprechen, in der zwei unterschiedliche Wissenschaftshaltungen nebeneinander herliefen, deren grundsätzliche Unvereinbarkeit zu einer seltsamen Uneinheitlichkeit auch in der staatlich-parteilichen Wissenschaftspolitik führten. Während beispielsweise in der fortbestehenden Akademie der Wissenschaften Marxisten und Nicht-Marxisten noch friedlich koexistierten, ging die SED - parallel zu ihrer Umwandlung in eine stalinistische Partei >Neuen Typs - an den Schulen und Universitäten seit 1948 daran, den marxistisch-leninistischen Monopolanspruch kompromißlos durchzusetzen.

Der aus dieser Umgestaltung resultierende Weg der akademischen Lehre in die Teilung der deutschen Geschichtsdisziplin machte sich in der historischen Forschung erst mit der Gründung beider deutscher Staaten 1949 deutlich bemerkbar. Dabei gingen die Distanzierungsbemühungen keineswegs allein von der östlichen Seite aus. Spätestens mit der steigenden Repression gegen die »bürgerlichen« Historiker im ostdeutschen Teilstaat verhärtete sich auch die 
Haltung des Verbandes der Historiker Deutschlands (VHD) gegenüber der ostdeutschen Fachentwicklung und setzte sich die resignierende Erkenntnis Ludwig Dehios durch, daß die Wissenschaft im anderen Teil Deutschlands "politischen Zwecken dienstbar « gemacht worden sei $^{3}$.

Erst von diesem Zeitpunkt an vermag das Begriffspaar von Konfrontation und Kooperation im Grunde die getrennte Entwicklung der deutschen Nachkriegshistoriographie adäquat zu erfassen. Der uneinheitlichen Entstehungsphase eines eigenständigen parteimarxistischen Geschichtsdiskurses in der frühen DDR, mindestens bis 1952/53, kann es nicht hinreichend gerecht werden, wie ein Blick auf die beiden ersten deutschen Historikertage nach dem Zweiten Weltkrieg lehrt, die 1949 in München und 1951 in Marburg stattfanden. In München waren unter den 231 Teilnehmern auch neun ostdeutsche Vertreter anwesend. Keiner unter ihnen allerdings repräsentierte eine eigenständige DDR-Geschichtswissenschaft, auch wenn der einzige Marxist unter ihnen, Walter Markov, seinen Standpunkt engagiert vertrat. Allerdings handelte auch er im Sinne seiner öffentlichen Wortmeldung von 1947, nach der niemand wünsche, »den historischen Materialismus für seine Unterdrückung in anderen Teilen Deutschlands durch ein Monopol in der Ostzone zu entschädigen ${ }^{4}$. München spiegelte daher nicht die Trennlinie zwischen Historikern West und Historikern Ost, sondern die schwächer werdende Abwehr einer in Grundfragen noch einheitlichen Historikerschaft gegen die Auswirkungen der politischen Teilung. Deutlicher zeigte sich zwei Jahre später, in der völligen Abwesenheit von DDR-Vertretern, auf dem Marburger Historikertag, wie die nicht-marxistische Geschichtswissenschaft in der DDR mittlerweile im Zuge des »Sturms auf die Festung Wissenschaft" an den Rand gedrängt worden und die Teilung vorangeschritten war. Dennoch bekannte sich noch ein Jahr später das erste Heft der neu gegründeten »Zeitschrift für Geschichtswissenschaft" ( $Z \mathrm{fG}$ ) zur Einheit der deutschen Geschichtswissenschaft und erklärte in einer programmatischen Vorbemerkung der Redaktion: »Der Kreis der Mitarbeiter an der >Zeitschrift für Geschichtswissenschaft، soll sich nicht auf Wissenschaftler beschränken, die sich zum Marxismus bekennen $\kappa^{5}$.

\footnotetext{
Ludwig DEHIO, Rezension, in: Historische Zeitschrift 177 (1954), S. 151 f.

Walter MARKOV, Historia docet?, in: Forum 1 (1947), S. 129.

Vorwort, in: ZfG 1 (1953), S. 3-6, hier S. 3. Daß es sich in dieser programmatischen Erklärung der ZfG-Redaktion nicht um bloße Rhetorik handelte, zeigen die intemen Diskussionen, die ihrer Formulierung vorausgingen; Bundesarchiv, Stiftung Archiv der Parteien und Massenorganisationen der DDR im Bundesarchiv [SAPMO-BArch], DY 30, IV $2 / 9.04 / 114$.
} 
II.

Ein weit unübersichtlicheres Bild bot zwei Jahre später der Historikertag in Bremen. Die angereisten DDR-Teilnehmer selbst trugen durch ihr unpolemisches Diskussionsverhalten dazu bei, dem Treffen den Charakter eines "gesamtdeutschen Gesprächs« zu geben. Die anschließende Polemik der ZfG aber gegen die "reaktionären und unwissenschaftlichen Bestrebungen « der westdeutschen Geschichtswissenschaft ${ }^{6}$ führte beinahe zwangsläufig zu dem westlichen Gegenvorwurf, daß in Wirklichkeit die ostdeutsche Disziplin durch den Druck der kommunistischen Machthaber ihres Wissenschaftscharakters beraubt worden sei. Der VHD selbst schwankte zwischen dem scharfen Protest gegen die "politische Diffamierung durch Umdeutung wissenschaftlich erarbeiteter Einsichten ${ }^{7}$ und der fortbestehenden Überzeugung, daß »wir [...] nichts tun dürfen, was den Zusammenhalt mit den Ost-Kollegen gefährden kann ${ }^{8}$. Der Historikertag von Ulm 1956 schien sogar die Hoffnung zu rechtfertigen, daß die sich abzeichnende Spaltung der deutschen Historikerschaft bald überwunden werden könne und die Einheit der Wissenschaft letztlich über die politischen Störeinflüsse der SED obsiege. Das moderate Diskussionsklima während der Tagung wie deren anschließende Würdigung in der ZfG zeugten von der Bereitschaft der östlichen Seite, anzuerkennen, daß zumindest »die jüngere Generation der westdeutschen Geschichtswissenschaft sich um ein objektives Bild der Geschichte bemüht«. Stärker noch mußte die Erwartung der Ost-Seite, $» d a ß$ die Auseinandersetzung zwischen Marxisten und Nichtmarxisten auf dem Gebiet der Geschichtswissenschaft trotz der prinzipiellen Verschiedenheit des Klassenstandpunktes beider Seiten nicht nur möglich, sondern fruchtbringend ist ${ }^{9}$, auch in der bundesdeutschen Zunft den Glauben nähren, daß die Einheit der historischen Disziplin in Deutschland trotz aller Irritationen fortbestehe oder sich zumindest erneuere.

Doch diese Hoffnung zerstob spätestens auf dem Historikertag von Trier 1958, als die westdeutsche VHD-Führung die sich steigernden Repressionen gegen nicht-marxistische Fachkollegen in der DDR zum Anlaß nahm, gegen die Hauptvertreter der DDR-Geschichtswissenschaft vorzugehen und ihnen als

6 Fritz KLEIN, Der 22. Deutsche Historikertag, in: ZfG 1 (1953), S. 905-908, hier S. 907.

7 In diesem Sinne argumentierte die - nicht verabschiedete - »Heppenheimer Erklärung«, mit der der Ausschuß des VHD sich 1954 gegen die Berichterstattung über den Bremer Historikertag in der ZfG zur Wehr setzte. Näheres bei Winfried SCHULZE, Deutsche Geschichtswissenschaft nach 1945, München 1989, S. 190.

8 Archiv des Verbandes der Historiker Deutschlands [AVHD] 6, Herbert Grundmann an Hermann Aubin, 17.4.1954.

9 23. Versammlung deutscher Historiker in Ulm, in: ZfG 5 (1957), S. 124-131, hier S. 127. 
„Gruppe unbedingt unerwünschter kommunistischer Historiker ${ }^{10}$ das Wort zu entziehen. Die DDR-Delegation machte den Vorgang zum Skandal, indem sie abreiste. Sie hinterließ zwei Protestnoten, die die Ausschlußerklärung des VHD als ein »Dokument der psychologischen Kriegführung« werteten, welches »erneut beweist, daß die behauptete >Einheit der Wissenschaft< eine Fiktion ist ${ }^{11}$.

Der ungeschickt wirkende Schritt des VHD war eine Reaktion auf die wachsende Repression der wenigen in der DDR verbliebenen NichtMarxisten, die aus ihrem Lehramt gedrängt wurden (wie Heinrich Sproemberg in Leipzig ${ }^{12}$ ), ihre Leitungspositionen räumen mußten (wie Fritz Hartung als Herausgeber der »Jahresberichte für deutsche Geschichte $«{ }^{13}$ ), nach ihrer $»$ Republikflucht« mit dem Entzug ihres akademischen Grades bedroht wurden (wie Irmgard Höß) oder gar den Freitod wählten (wie Karl Griewank, Martin Lintzel und Willy Flach). Unter dem Eindruck einer wissenschaftspolitischen Linie Ost-Berlins, die die sideologische Koexistenz gekündigt $^{14}$ und auf der III. Hochschulkonferenz im März 1958 den Marxismus-Leninismus zur verbindlichen Grundlage der Gesellschaftswissenschaften in der DDR erklärt hatte, gab der der VHD die Einheit der deutschen Geschichtswissenschaft öffentlichkeitswirksam für verloren und erklärte die in Trier ausgeschlossenen DDR-Historiker zu Fachvertretern, »mit denen wir die wissenschaftliche Gemeinschaft nicht weiter aufrechterhalten können ${ }^{15}$. Damit hatte sich in der westdeutschen Historikerzunft auch öffentlich ein Denkstil durchgesetzt, der die Entwicklung des Faches unter dem SED-Regime als Verfallsgeschichte begriff und als zunehmende ideologische Knebelung einer Wissenschaft, deren Bedeutung in eben dem Maße sank, in dem der politische Wille der Partei über die fachlichen Maximen der Geschichtsschreibung triumphierte ${ }^{16}$.

So die Formulierung von Gerhard Ritter, zitiert nach Franz WORSCHECH, Der Weg der deutschen Geschichtswissenschaft in die institutionelle Spaltung, 1945-1965, phil. Diss., Erlangen 1987, S. 201.

11 Zitiert nach: ibid., S. 218.

12 Veit DIDCZUNEIT, Heinrich Sproemberg - ein Außenseiter seines Faches. Unter besonderer Berücksichtigung seiner Tätigkeit als Leipziger Hochschullehrer 1950 bis 1958, in: DERS., Manfred UNGer, Matthias MIDDELL, Geschichtswissenschaft in Leipzig: Heinrich Sproemberg, Leipzig 1994, S. 11-90.

13 Den Vorgang behandelt WORSCHECH, Der Weg der deutschen Geschichtswissenschaft, (wie Anm. 10), S. 180ff.

14 Ernst HOFFMANN, Über Tendenzen, die den weiteren Fortschritt unserer Geschichtswissenschaft hemmen, in: Einheit. Zeitschrift für Theorie und Praxis des wissenschaftlichen Sozialismus 12 (1957), S. 1146-1163, hier S. 1150.

15 Erklärung des VHD, zit. nach SCHULZE, Die deutsche Geschichtswissenschaft (wie Anm. 7), S. 198.

16 Schon 1951 klagte der Leipziger Mediävist Heinrich Sproemberg gegenüber Gerhard Ritter: »Leider fehlen die Zeugnisse einer recht unfreundlichen Haltung aus den Histori- 
Im Binnendiskurs der DDR-Geschichtswissenschaft hingegen wurde der Eklat von Trier als willkommener Hebel eingesetzt, um die eigene Wissenschaft durch forcierte Abgrenzung von der Westkonkurrenz zu stabilisieren. "Allgemein ist unsere Arbeit in gesamtdeutschen Gremien (Hansischer Geschichtsverein u.a.) zu überprüfen«, forderte die Abteilung Wissenschaften im Zentralkomitee (ZK) der SED ${ }^{17}$. Auch die eigene Historikerschaft konnte unter Ausnutzung des Trier-Effekts zu größerer Einheitlichkeit veranlaßt werden. Man war sich über die Notwendigkeit einig, »bereits in den nächsten Wochen ein reges wissenschaftliches Leben in Form von öffentlichen Diskussionen, kleineren Tagungen usw. zu entfalten. Alle Institute sollen bis zur nächsten Woche mitteilen, was sie in dieser Beziehung vorhaben ${ }^{18}$. Die im Sommer 1958 noch 55 Historiker zählende Schar ostdeutscher Mitglieder des VHD trat noch im selben Jahr ausnahmslos aus dem Verband aus. Als zwei Jahre später der VHD zu seiner nächsten Tagung nach Duisburg lud, bewertete die ZfG das Programm des Duisburger Historikertages als »das raffinierteste, das je aufgestellt worden ist«. Es handele sich um die erste Tagung, »die unter der direkten Leitung des führenden NATO-Historikers Hans Rothfels, Tübingen, stattfindet«, und wie selbstverständlich nahmen DDR-Historiker an ihr nicht teil ${ }^{19}$.

Bis zu diesem Zeitpunkt verlief der Weg von der Teilung zur Konfrontation auf beiden Seiten parallel. Von östlicher Seite aus wurde der westlichen Historiographie in der Folge das Prädikat der Wissenschaftlichkeit verweigert und jeder »ideologischen Koexistenz« mit der Frage der Boden entzogen, »ob die Historiker die Geschichte [...] vom Standpunkt der aufsteigenden Arbeiterklasse oder vom Standpunkt der untergehenden Bourgeoisie betrachten, ob sie die reaktionäre Rolle des deutschen Imperialismus und Militarismus in der Geschichte entlarven oder verherrlichen $\aleph^{20}$. Scharf wurde 1957/58 der im Zuge des kurzzeitigen »Tauwetters « von 1956 besonders durch Jürgen Kuczynski propagierte Versuch zurückgewiesen, »in den prinzipiellen Fragen der Objektivität und Parteilichkeit gemeinsame Grundlagen der marxistischen und der bürgerlichen Geschichtsschreibung zu entdecken«. In den Diskursfiguren einer parteilichen Geschichtswissenschaft konnte historische Objektivität nur eine Funktion des Klassenkampfes darstellen: „Die Objektivität der wissenschaftlichen Geschichtsschreibung beruht jedoch auf dem Materialismus, auf dessen richtige Anwendung auf die geschichtlichen Erscheinungen ${ }^{21}$.

kerkreisen des Westens gegen uns nicht [...]. Wie schade ist es, daß der persönliche wie auch der wissenschaftliche Austausch gerade mit dem Westen so stark zurückgegangen ist «. AVHD 4, Heinrich Sproemberg an Gerhard Ritter, 23.11.1951.

17 SAPMO-BArch, DY 30/IV 2/9.04/151, Aktennotiz, 15.10.1958.

18 Ibid.

19 Ibid., Rolf Rudolph an das ZK der SED, Abt. Wissenschaften, 24.6.1962.

${ }^{20}$ HOFFMANN, Über Tendenzen (wie Anm. 14), S. 1154.

21 Ibid. »Durch die Konzeption der ideologischen Koexistenz mit der imperialistischen Geschichtsschreibung in Westdeutschland kann unsere Geschichtsschreibung nur ihr eigenes 
Damit war jede Verständigung von Historikern beider Lager unmöglich geworden. Sie mußte es auch sein, um nicht die künstliche Identität des sozialistischen Geschichtsdenkens in Frage zu stellen. In spiegelbildlicher Umkehrung wurde wiederum in der Bundesrepublik die in der DDR entstehende parteimarxistische Geschichtsschreibung aus der Fachdisziplin ausgeschlos$\operatorname{sen}^{22}$. Vertreter der "sowjetzonalen Geschichtsbetrachtung" konnten aus dieser Sicht »keine vollwertigen Wissenschaftler nach westlichen Begriffen ${ }^{23}$ sein, sondern verkörperten vielmehr den »Typ des Funktionärs [...], der ganz im Dienste der Staatspartei Geschichte schreibt $\aleph^{24}$. Aus der tradierten Geschichtswissenschaft war demnach unter Ulbricht »ein politisches und propagandistisches Mittel ersten Ranges « geworden, das »den Forderungen des Tages dienen und sich, ihres Wissenschaftscharakters entkleidet, mißbrauchen lassen $[\mathrm{mu} ß]$ wie selten zuvor ${ }^{25}$. Eine solche Forschung bedeutete keine wissenschaftliche Bereicherung, sondern kam vornehmlich als politische Gefahr in Betracht:

Gesicht verlieren. Ihre wissenschaftliche und politische Kraft schöpft unsere Geschichtsschreibung aus der Arbeiterklasse, deren grundlegende Klasseninteressen völlig mit der objektiven Wahrheit der geschichtlichen Entwicklung vom Kapitalismus zum Kommunismus übereinstimmen, aus dem Marxismus-Leninismus, der einzigen Lehre, die über eine wissenschaftliche Theorie und Methode für die Erkenntnis der menschlichen Geschichte verfügt, aus der Arbeiter-und-Bauern-Macht und ihrer führenden Kraft, der marxistisch-leninistischen Partei«; ibid., S. 1156.

Freilich blieben auch hier in der westdeutschen Historikerschaft unterschiedliche Nuancierungen durchaus bestehen, wie etwa Ritters briefliche Kritik an Dehios Ausführungen zur eben begründeten $\mathrm{ZFG}$ in der $\mathrm{HZ}$ anzeigte: „Im ganzen scheinen sie mir etwas matt. Ich bin nicht der Meinung, daß die `Zeitschrift für Geschichtswissenschaft‘ >ernsthaft gelesen zu werden verdient $<$ daß sie ,Anregungen wissenschaftlicher Natur enthält, daß sie ,Scharfsinn « zeigt, daß die 'geistige Eigenart und Kraft des Gegners` hervortritt, und daß die marxistische Doktrin heute noch als rerzieherische Aufrüttelung unserer Wissenschaft dienen kann. Außerdem bin ich nicht geneigt, den szeitgeschichtlichen Quellenwert ‘ der Berliner Schmierereien sehr hoch einzuschätzen und ärgere mich über so viele Verbeugungen vor diesen Berliner Gesinnungslumpen«; AVHD 7, Gerhard Ritter an Hermann Aubin, 23.9.(?)1954.

Fritz KOPP, Die Wendung zur »nationalen« Geschichtsbetrachtung in der Sowjetzone, München ${ }^{2} 1955$, S. 6.

24

Reinhart BECK, Die Geschichte der Weimarer Republik im Spiegel der sowjetzonalen Geschichtsschreibung, Bonn, Berlin 1965, S. 66. Im Vorfeld des Internationalen Historikertags in Rom 1955 äußerte der VHD-Vorsitzende sich besorgt, wwelchen Eindruck wir den Ausländern vermitteln, wenn eine solche Zahl von sogenannten Historikern der Ostzone aufmarschiert und ihnen gegenüber Deutschland repräsentiert. Wir tun damit diesen Genossen das an, worauf alle ihre Politik derzeit gerichtet ist: Wir machen sie hoffähig, wir stellen ihnen das Zeugnis aus, daß wir sie wissenschaftlich für gleichwertig halten, während ihre Haltung nach dem Bremer Kongresse deutlich bewiesen hat, daß sie mit aller Geschichtskenntnis nur Politik treiben«; AVHD 7, Hermann Aubin an Walther Holtzmann, 7.8.1955. Albrecht TIMM, Das Fach Geschichte in Forschung und Lehre in der Sowjetischen Besatzungszone seit 1945, Bonn, Berlin ${ }^{3} 1961$, S. 7. 
Man mag das Niveau dieser scheinwissenschaftlichen Schriften einschätzen wie man will, politisch gewichtig sind sie auf alle Fälle, denn die darin vorgetragenen Auffassungen haben eine zwar primitive, aber geschlossene und blendende Scheinlogik in sich, die auf gewisse Leserkreise durchaus wirken kann $^{26}$.

Die verfallsgeschichtliche Betrachtungsweise klammerte die ostdeutsche Historiographie als "pseudowissenschaftlich ${ }^{27}$ aus dem fachlichen Diskurs aus und ordnete die Beschäftigung mit ihr in den Kontext antitotalitärer Gefahrenabwehr ein: »Bei der Auseinandersetzung mit dem totalen Bolschewismus, die auf allen Ebenen erfolgt, darf kein entscheidendes Gebiet übersehen werden ${ }^{28}$.

Die aus dieser Zeit stammenden Verlautbarungen zum Charakter des anderen Lagers beschränkten sich daher auf beiden Seiten vor allem auf Enthüllungsschriften und Kampfansagen. Besonders martialisch verfuhr hier die DDR-Seite, die Ende der 1950er Jahre bei offiziösen Historikerberatungen die SED-Historikerschaft auf ein Zerrbild ihrer westdeutschen Konkurrenz einschwur. Es trug Züge einer von den Machthabern gegängelten Afterwissenschaft, die ihr selbst vom Westen aus immer wieder vorgehalten wurden: »Dort haben wir ein von oben zusammengezimmertes und dirigiertes Kollektiv von Historikern, das in wissenschaftlichen Zeitschriften und Büchern, in Zeitungen, im Rundfunk und überall tagtäglich reagiert, sämtliche Wege nutzt, um historiographisch den deutschen Imperialismus bei der Vorbereitung des Atomkrieges zu unterstützen. Das ist doch die Sache. Und wir, die wir doch nicht von diesen Widersprüchen zerrissen sind, wo doch unsere wissenschaftliche Arbeit völlig mit der objektiven Wahrheit des historischen Fortschritts übereinstimmt, wir tun es nicht [...]. Das heißt, wir haben ein Klassenbewußtsein, das weniger entwickelt ist als das politische Klassenbewußtsein der monopolkapitalistischen Geschichtsschreiber drüben«, rief ein führender SEDHistoriker im Dezember 1958 auf einer von der Parteispitze anberaumten »Historikerberatung « aus und wurde von Ulbricht selbst unterstützt, der unter dem Beifall der Anwesenden erklärte:

Es gibt dort eine einheitlich ideologisch-politische Leitung der gesamten Geschichtsforschung. [...] Die Geschichtsschreibung Westdeutschlands ist auf die Durchfuhrung des psychologischen Krieges abgestellt und darauf, daß im Jahre 1961 die Rüstung der westdeutschen NATO-Truppen fertig ist, und bis zu dieser Zeit muß die entsprechende ideologische Verseuchung in Westdeutschland erreicht sein. Das ist dort exakt ausgearbeitet ${ }^{29}$.

In der Bundesrepublik hingegen fanden insbesondere Erfahrungsberichte geflüchteter Fachkollegen weite Verbreitung, die mit der Legitimation der eige-

26 KoPP, Die Wendung (wie Anm. 23), S. 6f.

27 Walther HOFER, Geschichtsschreibung als Instrument totalitärer Politik, in: DERS. (Hg.), Wissenschaft im totalen Staat, München 1964, S. 198-227, hier S. 206.

${ }^{28}$ KOPP, Die Wendung (wie Anm. 23), S. 7.

29 SAPMO-BArch, DY 30/IV 2/1.01/392, Stenographische Niederschrift der Abteilung Wissenschaften beim ZK mit Genossen Historikern im Großen Sitzungssaal des Zentralhauses der Einheit am Mittwoch, dem 17. Dezember 1958. 
nen Leiderfahrung über die Entwicklung der ostdeutschen Geschichtsschreibung aufklären wollten ${ }^{30}$. Durchgängig beschrieben sie die "Sowjetisierung des Geschichtsbildes in Mitteldeutschland « als einen Niedergangprozeß in einzelnen »Etappen ${ }^{31}$, dessen Schlußpunkt die gänzliche Instrumentalisierung der "parteihörigen" Wissenschaft bildete und damit die "Aufspaltung des deutschen Geschichtsbildes in ein westliches und ein östliches « ${ }^{32}$. Aus dieser Sicht hatte jede innerwissenschaftliche Verständigungsmöglichkeit ihr Ende gefunden: "Zwischen West und Ost jedoch scheint auch auf dem Boden einer fachwissenschaftlichen Auseinandersetzung kein echtes Gespräch mehr möglich $\aleph^{33}$.

Es war daher nur folgerichtig, daß die Literatur zur DDR-Historiographie sich bis zur Mitte der 1960er Jahre vornehmlich bemühte, Unstimmigkeiten und Widersprüche zwischen wissenschaftlichem Anspruch und politisch gelenkter Realität aufzudecken: "Die Grenze zwischen Wissenschaft und Propaganda wird weitgehend verwischt«, resümierte Jürgen von Hehn schon $1954^{34}$, und Georg von Rauch kommentierte ein Jahr später: "Es wird in Moskau beschlossen, welche Maßstäbe jeweils zu gelten haben und ob sie [...] auch den Satelliten zustehen ${ }^{35}$. Der so organisierte, ausgrenzende Betrachtungsansatz fokussierte auf die »nicht zu übersehende[n] Lücken ${ }^{36}$; er insistierte auf den "zahlreiche[n] weiße[n] Flecken ${ }^{37}$ und tadelte »die offensichtliche Ungleich-

Erfahrungsberichte von in den Westen gewechselten Historikem standen freilich vor dem paradoxen Umstand, ihre höhere Authentizität mit einer in gleichem Maße sinkenden Glaubwürdigkeit bezahlen zu müssen, hatten sie doch im Berichtszeitraum in eben den Verhältnissen gewirkt, über die sie warnend aufklären wollten. Der in die Bundesrepublik geflüchtete Albrecht Timm, der von 1952 bis zu seiner Flucht im August 1955 eine auBerplanmäßige Professur für mittelalterliche Geschichte an der Berliner HumboldtUniversität bekleidet hatte, löste dieses Problem als Verfasser einer Publikation über "Das Fach Geschichte in Forschung und Lehre in der Sowjetischen Besatzungszone seit 1945« so: »Die hier vorgelegte Untersuchung [...] schöpft aus dem eigenen Erleben und aus Beobachtungen während eines Jahrzehnts praktischer Tätigkeit in der Sowjetzone, wo der Verfasser zwar Auseinandersetzungen mit dem Marxismus-Leninismus nicht vermied, von dessen Interpreten aber stets als 'Nichtmarxist` und `Bürgerlicher` gekennzeichnet wurde« (S. 5).

31 Jürgen VON HEHN, Die Sowjetisierung des Geschichtsbildes in Mitteldeutschland, in: Europa-Archiv 19 (1954), S. 6929-6938 und 6973-6977, hier S. 6929f.

32 Georg vON RAUCH, Das Geschichtsbild der Sowjetzone, in: Jahrbuch der RankeGesellschaft 1954, Frankfurt a.M., Berlin, Bonn 1955, S. 101-119, hier S. 101.

33 Ibid.

34 VON HEHN, Die Sowjetisierung des Geschichtsbildes (wie Anm. 31), S. 6976.

35 VON RAUCH, Das Geschichtsbild der Sowjetzone (wie Anm. 32), S. 108.

${ }^{36}$ BECK, Die Geschichte der Weimarer Republik (wie Anm. 24), S. 20.

37 Ibid., S. 21. Vgl. zu "weißen Flecken« in der kommunistischen Geschichtsschreibung insbesondere Hermann WEBER, Ulbricht fälscht Geschichte, Köln 1964; DERS., "Weiße Flecken" in der DDR-Geschichtsschreibung, in: Aus Politik und Zeitgeschichte B 11/1990, S. 3-15; DERS., »Weiße Flecken« in der Geschichte. Die KPD-Opfer der Stalinschen Säuberungen und ihre Rehabilitierung, Frankfurt a.M. 1990. 
mäßigkeit, mit der in Mitteldeutschland bis jetzt die Geschichte der Weimarer Republik erforscht wurde $\aleph^{38}$. Die Leistungskraft dieses lange dominanten Paradigmas lag in der Aufmerksamkeit für die genuine Verschiedenheit der beiden deutschen Geschichtswissenschaften und die politische Abhängigkeit einer »unfreien, völlig dirigierten Geschichtsschreibung, die sich als Mittel im Kampf um die totale kommunistische Welteroberung versteht ${ }^{39}$.

\section{III.}

Ziehen wir ein erstes Fazit: Eine Beziehungsgeschichte der beiden deutschen Historiographien läßt sich erst ab der Zeit ihrer ausgebildeten Teilung ab etwa Mitte der 1950er Jahre schreiben. Für die folgenden zehn Jahre deckt der Begriff Konfrontation das wechselseitig ausgrenzende Handeln beider Seiten zureichend ab. Zu erörtern bleibt, ob das gleiche in Bezug auf die 1970er und 1980er Jahre für den Komplementärbegriff der $»$ Kooperation« gilt.

Leicht fällt die Antwort für die westdeutsche Seite, die in der Tat mit dem Generations- und Paradigmenwechsel in den 1960er und 1970er Jahren den Weg von der ausgrenzenden Konfrontation zur einbeziehenden Kooperation ging. In den 1970er Jahren begann die bisher normativ ablehnende Rezeptionshaltung zugunsten eines pragmatischen Deutungsmusters in den Hintergrund zu treten, das die einzelnen Forschungsfelder der östlichen Historiographie nach dem Grad ihrer politischen Belastung und Formung zu differenzieren bereit war. Aus dieser neuen Perspektive stellte sich die bis dahin als totalitäre Wüste konturierte Wissenschaftslandschaft östlich der Elbe nun als ein schwer überschaubares Gelände mit überraschenden Ausblicken dar, das mancherorts zum Horizont hin offen schien und zur Kooperation auf vielen Feldern einlud, sofern sie nur abseits von den Kernstücken der sozialistischen Herrschaftslegitimation lagen.

Die voranschreitende Einpassung der DDR-Historiographie in die »Ökumene der Historiker" eröffnete neue Aussichten auf bisher unbeachtete Gemeinsamkeiten und Wechselwirkungen der beiden deutschen Geschichtswissenschaften. Schon Werner Conze konstatierte 1977 eine Annäherung von Forschungsinteressen und Fragestellungen im Zuge der innerwissenschaftlichen Traditionskritik der 1960er Jahre und des neu erwachten sozialhistorischen Interesses ${ }^{40}$. Eine »erstaunliche Parallelität« beider Historio-

40 Werner CONZE, Die deutsche Geschichtswissenschaft seit 1945. Bedingungen und Ergebnisse, in: Historische Zeitschrift 225 (1977), S. 1-28, S. 25, 28. 
graphien in ihrer Grundlagenkrise, in ihren Paradigmenwechseln und in ihren Leitbildern stellte Heydemann in seiner vergleichenden Dissertation über die "Geschichtswissenschaft im geteilten Deutschland « fest ${ }^{4 !}$. Aufgrund dieser Entwicklung verlor das Bild einer klaren Hierarchie zwischen pluraler und gelenkter Wissenschaft in den 1970er und 1980er Jahren allmählich seine Konturen und machte einer Vorstellung tendenzieller Gleichwertigkeit Platz. Auch bundesdeutsche Historiker meinten bereits seit Anfang der 1970er Jahre mehr und mehr einräumen zu müssen, daß die Standortgebundenheit jeder Forschung geltender Minimalkonsens auf beiden Seiten sein müsse und das Ideal des freien Forschers auch im Westen nicht mehr haltbar sei. Ende der 1980er Jahre begann dann sogar der östliche Vorhalt, »daß Parteilichkeit ein Merkmal auch der bundesdeutschen Historiographie sei«, im Westen Widerhall zu finden ${ }^{42}$. So schienen am Vorabend der revolutionären Wende von 1989 die gestiegene internationale Anerkennung und Beweglichkeit der DDRGeschichtswissenschaft wie die gewachsene Öffnungsbereitschaft der westdeutschen Gegenseite den Weg zu einer schrittweisen Vereinigung der geteilten Historiographie vorzuzeichnen.

IV.

$\mathrm{Da} ß$ diese längst nicht von allen westdeutschen Fachrichtungen und -schulen geteilte Bereitschaft zur inhaltlichen Auseinandersetzung von der DDR-Seite im Rahmen des historischen Herrschaftsdiskurses eher als Identitätsproblem statt als Handlungschance erfahren werden solite, zeigte sich schon früh. Insbesondere die Kontroverse um Fritz Fischers »Griff nach der Weltmacht« weitete sich zum Aufbruch einer jüngeren bundesdeutschen Historikergeneration zu neuen sozialwissenschaftlichen Ufern aus, die sozioökonomischen Herangehensweisen an die Geschichte weit weniger distanziert gegenüberstand als die Gründergeneration der deutschen Nachkriegshistoriographie. Öffentliche Wirkung erregte dieser Trendwechsel auf dem Berliner Historikertag 1964, der schon im Vorfeld erhebliche Anstrengungen der für die Historie zuständigen Lenkungsorgane der SED auslöste. Auf Drängen der »Ideologischen Kommission « und Kurt Hagers persönlich wurde ein »Maßnahmeplan über unsere Aktionen« ausgearbeitet, der zunächst als Ausdruck eigener Stärke

41 Günther HEYDEMANN, Geschichtswissenschaft im geteilten Deutschland. Entwicklungsgeschichte, Organisationsstruktur, Funktionen, Theorie- und Methodenprobleme in der Bundesrepublik Deutschland und in der DDR, Frankfurt a.M. 1980, S. 246.

42 Dietrich STARITZ, DDR-Geschichte im deutsch-deutschen Wissenschaftsdialog, in: Aus Politik und Zeitgeschichte B 34/1989, S. 10-17, hier S. 17. 
verbuchte, daß »sich die bürgerliche westdeutsche Geschichtswissenschaft unter dem Einfluß der marxistischen Geschichtswissenschaft der DDR immer stärker mit der Geschichte der Arbeiterbewegung beschäftigen muß«. Vor diesem Hintergrund wurde die "Benennung von Genossen, die als offizielle Beobachter am Westberliner Kongreß teilnehmen«, ebenso für sinnvoll erachtet wie eine ganze Serie von Briefen, Interviews, Artikeln und Rundfunkvorträgen, mit denen die SED-Historiker ihre Stellung wahren und auf ihre westlichen Kollegen einzuwirken gedachten ${ }^{43}$.

Sorgfältig wurde allerdings bei allen Schritten darauf geachtet, daß die vorsichtige Wiederaufnahme einer Dialogpolitik nicht auf den Stand von Ulm zurückfiel und die Grenzen zwischen zwei gegensätzlichen Wissenschaften verwischte:

Die Auswahl [der nach West-Berlin entsandten Beobachter, M.S.] ist erstens nach dem Gesichtspunkt der politischen Zuverlässigkeit, zweitens unter Berücksichtigung der fachlichen Qualifikation und drittens unter Beachtung des Grundsatzes erfolgt, da 9 die Anwesenheit dieser Historiker aus den Kreisen des wissenschaftlichen Nachwuchses nicht als eine offizielle Anerkennung des Kongresses gewertet werden kann ${ }^{44}$.

Am Rande des Historikertages ergab sich sogar ein geheimes Treffen aufgeschlossener Nachwuchshistoriker der westlichen Seite mit ausgewählten Ost-Kollegen über »Methodologische Probleme der Weltkriegsforschung«. Der anschließend erstattete Bericht versuchte, das zarte Kooperationspflänzchen zu hegen:

Es wurde deutlich, daß die qualifizierten Historiker der DDR die Auseinandersetzung nicht zu fürchten brauchen und sich in den letzten Jahren Voraussetzungen schufen, faktisch wie theoretisch offensiv aufzutreten [...]. Unseres Erachtens sollte die Diskussion weitergeführt werden, wobei wir darauf drängen sollten, daß eine Gruppe von Genossen zu einem Gespräch nach Westdeutschland - wiederum in einem beschränkten Fachkreis - eingeladen wird ${ }^{45}$.

Dazu sollte es allerdings nicht mehr kommen. Wenige Monate später wies eine vertrauliche "Information an den Genossen Hager« aus der Abteilung Wissenschaften darauf hin, daß es seit dem Berliner Historikerkongre $\beta$ am Akademie-Institut für Geschichte zu heftigen Diskussionen über die gegenwärtige westdeutsche Geschichtsschreibung gekommen sei. Eine Gruppe von Neuzeithistorikern habe die »Erweiterung von 'Kontakten< auch zu solchen Historikern gefordert, die uns ideologisch und politisch als Gegner bekannt sind ${ }^{46}$. Die sich am Akademie-Institut hinziehenden Auseinandersetzungen

43 Archiv der Berlin-Brandenburgischen Akademie der Wissenschaften [ABBAW], Zentralinstitut für Geschichte [ZIG], 165, Ernst Engelberg an Johannes Hörnig, 25.6.1964.

44 Ibid.

45 Ibid., 334, Kurt Pätzold, Bericht über einen Diskussionsabend mit westdeutschen Historikern am 9. Oktober 1964 in der Akademie-Zentrale (Erste Fassung, 10.10.1964).

46 Ibid., Ernst Engelberg Diskussionsbeitrag in der Mitgliederversammlung der SEDGrundorganisation des Instituts für Geschichte der DAW, 15.6.1965. 
um Abbruch oder Fortführung der innerdeutschen Kontakte wurden schließlich mit einem Machtwort des Institutsdirektors beendet, der davor warnte, daß gerade die "Tauben" in der westdeutschen Geschichtswissenschaft »einiges tun [können], um die innere Geschlossenheit der Genossen Historiker [...] und ihre Verbundenheit mit der Gesamtpartei zu lockern ${ }^{47}$. Ein schon vereinbartes Folgetreffen mit westdeutschen Historikern in Kiel wurde abgesagt, und fortan gaben DDR-Historiker auf Anweisung etwa noch bestehende Mitgliedschaften in westdeutschen Fachverbänden zurück. Die Kontakte zwischen den verfeindeten Lagern gingen in dieser Zeit kaum über Begegnungen auf internationalen Konferenzen und wechselseitige Archivbesuche hinaus, und sie wurden von seiten der DDR durch eine restriktive Genehmigungspraxis gegenüber auswärtigen Benutzungsanträgen ebenso gesteuert wie in der Einschränkung eigener Außenverbindungen auf einen »ideologisch gefestigten« Stamm von $»$ Reisekadern $\lll$.

Fortan lauteten routinemäßige Meldungen über »Beziehungen $[\ldots]$ zu Einrichtungen und Personen in der BRD« gewöhnlich so:

Das ZI [Zentralinstitut] für Geschichte ist an der Benutzung von Archiven und Bibliotheken in der BRD und in Westberlin [...] interessiert. Aus Archivbenutzung und Anforderung von Materialien auf dem Postwege ergeben sich sowohl schriftliche als auch persönliche Kontakte mit den Archiv- bzw. Bibliotheksleitungen und -verwaltungen bzw. einzelnen Angestellten. Eine wissenschaftliche Zusammenarbeit findet jedoch nicht statt ${ }^{48}$.

Innerdeutsche Begegnungen außerhalb der von DDR-Seite mit ausgewählten Delegationen beschickten Internationalen Historikerkongresse hatten in dieser Zeit Seltenheitswert und tauchten in den Rechenschaftsberichten historischer Forschungseinrichtungen vor allem als Prüfung der eigenen Standhaftigkeit gegenüber der lockenden Herausforderung durch die »bürgerliche" $\mathrm{Ge}$ schichtswissenschaft auf, wie der Jahresbericht des Zentralinstituts für Geschichte für 1978 ausweist: „Gelegentlich gab es von seiten der BRD Versuche, sogenannte sinnerdeutsche` Kontakte anzuknüpfen, die alle zurückgewiesen wurden $\alpha^{49}$.

Doch im Zuge des weltweiten Entspannungsprozesses und des unter Honecker forcierten Strebens nach internationaler Anerkennung ließ sich das Konzept einer Normalisierung durch Nihilierung nicht länger aufrechterhalten. Die entscheidende Voraussetzung begann ihm immer weiter zu entgleiten: die gesellschaftliche und politische Abschließung des zweiten deutschen Staates. Eine ostdeutsche Geschichtswissenschaft, die unermüdlich um die Aufnahme in den Internationalen Historikerverband, das CISH, kämpfte, konnte auf Dauer um so weniger in der Abschottung verharren, als das westliche Werben um Kontaktaufnahme ihr in der Zeit der Détente selbst nicht zuletzt als der beste

47 Ibid.

48 ABBAW, ZIG 174, Meldung vom 7.6.1977, Anlage.

49 ABBAW, ZIG 088. 
Beleg ihrer immer deutlicheren Anerkennung und Überlegenheit erscheinen mußte ${ }^{50}$. $"$ Derartige Angebote sollten beachtet und geprüft werden. Die Geschichtswissenschaft der DDR besitzt die Kraft, solche Dialoge im Sinne unserer ideologischen Offensive $\mathrm{zu}$ führen ${ }^{51}$, erläuterte die HistorikerGesellschaft der DDR 1984 ihre Empfehlung, auf einen von Lutz Niethammer und Hans Mommsen ausgehenden Dialogvorschlag zur Zeitgeschichte einzugehen. Auch die eigene Historikerschaft drängte immer stärker auf erleichterte Reise- und Austauschmöglichkeiten mit dem Westen und konnte sich hierbei obendrein einer systemkonformen Argumentation bedienen:

Da Beweiskraft und Wirksamkeit und damit die internationale Geltung unserer geschichtswissenschaftlichen Forschungsergebnisse in hohem Maße von der Erschließung neuen Quellenmaterials bestimmt werden, ist die optimale Nutzung der Möglichkeiten zu Archiv- und Bibliotheksstudien auch in nichtsozialistischen Ländern [...] unverzichtbar ${ }^{52}$.

Freilich wurden auf den Kommandohöhen der Parteiwissenschaft sorgfältig Kosten und Nutzen der lockenden Kooperation mit dem Klassenfeind abgewogen, die in den 1980er Jahren zu einer lawinenartigen Zunahme von genehmigten West-Reisen und Austauschbeziehungen führte:

Es häufen sich Einladungen zu Vorträgen, Tagungen und zu gemeinsamen Veröffentlichungen, besonders seit Abschluß des Kulturabkommens DDR-BRD. Realisiert werden vorrangig diejenigen Vorschläge, die der DDR bedeutenden wissenschaftlichen und politischen (z.T. auch finanziellen) Nutzen bringen und die einer weiteren fruchtbaren Zusammenarbeit mit uns nahestehenden linksorientierten, liberalen sowie einem Dialog zugänglichen realistischen bürgerlichen Historikern dienen ${ }^{53}$.

Doch diese Kanalisierungsbemühungen erwiesen sich als nutzlos. Rasch verschwanden aus den Reiseberichten derselben Zeit die identitätsverbürgende

50 Als Beispiel sei die Argumentation des ostdeutschen Handbuchs »Kritik der bürgerlichen Geschichtsschreibung « angeführt: „Der marxistisch-leninistischen Forschung wird [...] von der bundesrepublikanischen Historie zunehmend Aufmerksamkeit gewidmet. In der Tat unterscheiden sich Arbeiten und Rezensionen über die Geschichtswissenschaft der DDR und einzelne ihrer Ergebnisse, die in den letzten Jahren erschienen sind, nicht unbeträchtlich von jenen Auslassungen, die in den fünfziger Jahren und noch zu Beginn der sechziger Jahre das Feld beherrschten. Eine Ursache dafür kann darin erblickt werden, daß sich die Geschichtswissenschaft der DDR ebenso entfaltete und internationale Geltung gewann wie der sozialistische Staat, mit dem sie aufs engste verbunden ist. Daraus ergab sich auch, daß eine Geschichtswissenschaft nicht länger ignorantenhaft behandelt werden kann, von der befürchtet wird, >das Bild von der politischen Geschichte Deutschlands im 19. und 20. Jahrhundert im Ausland, werde sin einigen Jahrenc von ihr bestimmt sein«; Werner BERTHOLD u.a. (Hg.), Kritik der bürgerlichen Geschichtsschreibung. Handbuch, Köln ${ }^{4} 1977$, S.134 mit Bezug auf eine Aussage Dieter Riesenbergers.

51 Der Bundesbeauftragte für die Akten des Staatssicherheitsdienstes der ehemaligen DDR, Zentralarchiv, AGMS 5397/85, Zusammenfassung eines Berichtes der Historikergesellschaft der DDR, 10.4.1984.

52 ABBAW, ZIG 686, Bd. 2, Bericht über die Leistungsentwicklung und die Erfüllung der anteiligen Aufgaben des Zentralen Forschungsplanes [...] im Jahre 1986, 10.12.1986.

53 ABBAW, ZIG 163/1, Jahresbericht des ZIG für 1986. 
Betonung der eigenen Parteilichkeit und die kategorische Abgrenzung vom bürgerlichen Gegner zugunsten einer nicht weniger stereotypen Beteuerung des beiderseitigen Nutzens einer fachlichen Verständigung im Zeichen des bedrohten Weltfriedens. Nach ihrer Rückkehr berichteten DDR-Historiker nun über »sehr objektiv gehaltene und neue Forschungsergebnisse« ihrer westlichen Kollegen, beklagten die Schwierigkeiten, in einen »beiderseits aufgeschlossenen Dialog« über die Kriterien geschichtswissenschaftlicher Erkenntnis $\mathrm{zu}$ kommen, und empfanden ihre Teilnahme gleichwohl als "zweckmäßig und nützlich, da die Gelegenheit besteht, den marxistischen Standpunkt [...] offensiv darzulegen«, und »von allen Seiten das Interesse an weiterer Zusammenarbeit mit der DDR-Historiographie geäußert « werde ${ }^{54}$.

Die Integration von immer mehr historischen Forschungsfeldern der ostdeutschen Fachwissenschaft wurde erleichtert durch eine deutlichere Bereitschaft der westdeutschen Historiographie, ihre Wende von der normativen Ausgrenzung zur pragmatischen Einbeziehung des ostdeutschen Schwesterfachs bis an den Rand der eigenen Identitätsaufgabe weiterzutreiben. Im bundesdeutschen Verständnis hatte die DDR-Geschichtswissenschaft auf ihrem Weg der Emanzipation von politischer Bevormundung durch die SED ein tüchtiges Stück zurückgelegt und war in ihre »Verwissenschaftlichungsphase« eingetreten, die sie von einem selektiven zu einem integralen Geschichtsbild geführt habe ${ }^{55}$. Selbst eine administrative Entscheidung der DDR-Wissenschaftspolitik wie die Einführung des Rates für Geschichtswissenschaft im Jahre 1968 diente aus dieser Sicht zum Nachweis, daß »das Prinzip bisheriger Dekretierung politisch-ideologischer Aufgaben der Geschichtswissenschaft durch die SED von einem konsultativen Kommunikationsprozeß abgelöst« worden $\mathrm{sei}^{56}$.

Die Folgen dieser beiderseitigen Öffnung allerdings waren für alle Beteiligten unvorhergesehen: Die unterstützende Kooperationsbereitschaft des Westens entpuppte sich schließlich als identitätszerstörender Konkurrenzdruck für den Osten. Als beispielsweise im Oktober 1987 das die historische Forschung in der DDR dominierende Akademie-Institut für Geschichte auf einer Klausurtagung Bilanz über seine Stellung im internationalen Wettbewerb zog, war man sich einig, daß das bisherige Profil erhalten und gleichzeitig ausgebaut werden müsse:

54 ABBAW, A 8485, Wolfgang Küttler, Konferenz »Theorie der Geschichte«, Besprechung mit Prof. Kocka, 9.-12.12.1987, und Dietrich Eichholtz, Sofortbericht über die Reise nach Bochum (Vortragsreise) und nach Brüssel (Studienreise) in der Zeit vom 10. bis 23.11.1987.

5s Alexander FISCHER, Günther HEYDEMANN, Weg und Wandel der Geschichtswissenschaft und des Geschichtsverständnisses in der SBZ/DDR seit 1945, in: DIES. (Hg.), Geschichtswissenschaft in der DDR, Bd. 1: Historische Entwicklung, Theoriediskussion und Geschichtsdidaktik, Berlin 1988, S. 3-30, hier S. 19.

Ibid., S. 15. 
Zugleich sind mit der Sozialgeschichte, Alltagsgeschichte, Proletariatsforschung in der Volkskunde, Regionalgeschichte, Kirchengeschichte, Akademiegeschichte neue Forschungsrichtungen zu entwickeln bzw. weiterzuentwickeln, die Forschungen zur Geschichte der DDR auszubauen, die geschichtswissenschaftliche Information auf ein höheres Niveau zu heben und weitgehend auf EDV umzustellen und weitere Forschungslücken zu schließen. ${ }^{57}$

Deutlicher noch beschrieb der Direktor dieses Instituts die Sprengkraft, die aus einer ungeschützten Konkurrenz zwischen den beiden deutschen Geschichtswissenschaften resultieren müsse, in einer Dienstbesprechung seines Hauses über ein zum Thema »Erbeerschließung und Traditionspflege« vorgelegtes Arbeitspapier:

Prof. Schmidt antwortet, daß er ursprünglich das Papier wieder einziehen wollte, es aber jetzt doch in den Händen der Bereichsleiter läßt. Er bat aber darum, vor Überarbeitung des Papiers noch keine institutsweite Diskussion zu beginnen, es nur für den eigenen Gebrauch zu nutzen und evtl. einen bestimmten Kreis von Mitarbeitern einzubeziehen [...]. Im Herbst wird dann zur ganzen Problematik ein Institutsplenum stattfinden, auf dem dann von uns verantwortungsbewußt alle Fragen in der ganzen Breite ins Institut getragen werden können. Dann ist zu überlegen, ob und was veröffentlicht werden kann. Das wird auch von der Großwetterlage abhängig sein, denn jetzt ist noch nicht abzusehen, wie die ganze Erbe- $/$ Traditionsproblematik in den nächsten fünf Jahren angegangen wird. Auf jeden Fall geht es aber nicht mehr so weiter, daß wir weiter differenzieren und breiter werden bei allen anderen Klassen und Schichten, nur bei der Führungskraft der sozialistischen Gesellschaft nicht. Wenn dort nicht nachgezogen wird, müssen wir bremsen, denn das ist dem Gegner schon so auffällig, daß es mich eigentlich wundert, daß er höflich, freundlich und zuvorkommend ist und uns das nicht tagtäglich um die Ohren haut. Alle Arbeiten von ihnen haben diesen Fragenkomplex noch nicht angepackt. Nur in persönlichen Gesprächen haben sich westdeutsche Vertreter geäußert und ganz brutal auf diesen offenkundigen Rückstand hingewiesen. Und dieser Rückstand liegt nicht bei uns. Weiter, als hier zur Arbeiterklasse Stellung genommen ist, möchte Prof. Schmidt nicht gehen. Mehr können wir dazu nicht sagen, das müssen andere, dazu Berufene, tun. Von uns ist im Papier auf die Gefahr und daß Disproportionen und Unstimmigkeiten im Geschichtsbild, was ich ausdrücklich betone, entstehen können, hingewiesen worden $^{58}$.

Offener als je zuvor monierten DDR-Historiker nun mit Blick auf die deutschdeutsche Konkurrenz die eigene restriktive Forschungspraxis oder, schlimmer noch, mahnten sie tabubrechende Einschätzungen auch in der Zeitgeschichte an, um nicht vom Gegner überholt zu werden. Immer häufiger bewerteten interne Fachgutachten, die Voraussetzung einer Druckfreigabe waren, vorgelegte Arbeiten nach den Kriterien von Komplexität und Ausgewogenheit und wiesen tadelnd auf »Vereinfachungen« und »Einseitigkeiten« hin. Eine besondere Konjunktur erlebte in diesem Kontext die Forderung nach Differenzierung, die eine Brücke vom heimischen zum internationalen Fachverständnis zu schlagen erlaubte und in den 1980er Jahren eine geradezu epidemische Verbreitung in der internen Fachpraxis erlebte. Welche identitäts-

57 ABBAW, ZIG 091/6, Diskussionsprotokoll zum Tagesordnungspunkt 1 der Dienstbesprechung vom 15.5.1985.

58 Ibid. 
zerstörenden Folgen für das sozialistische Geschichtsdenken aus seiner allmählichen Integration in die communauté scientifique erwuchsen, illustriert das Ergebnis einer west-östlichen Diskussion über einen von der DDR-Seite für die Fernuniversität Hagen erarbeiteten Lehrbrief zur Sozialgeschichte. Wie der entsprechende Reisebericht festhielt, problematisierten die Hagener Kollegen nicht nur das Dogma der einheitlichen revolutionären Umgestaltung in der SBZ und die vom Autor behauptete Einheit von Wirtschafts- und Sozialpolitik in der DDR, sondern auch den von ihm verwendeten Klassenbegriff und die geringe Komplexität seines Verständnisses von gesellschaftlichem Fortschritt oder von Arbeiterinteressen:

In der Diskussion zeigten sich beträchtliche Verständigungsschwierigkeiten [...], und schon aus Zeitmangel wurde von unserer Seite mehr postuliert, als sich nachweisen und erläutern ließ [...]. Obwohl die Diskussion nicht recht befriedigen konnte, erfullte sie insofern ihren Zweck, als sie [...] uns anzeigte, wo wir unser Anliegen besser verständlich machen und sverkaufen müssen $^{59}$.

Das gesamtdeutsche Beziehungsschauspiel endete schließlich in einem doppelten Mißverständnis. Im Westen verstand man die angestrebte Normalisierung durch Kooperation als Beistand zur Verwissenschaftlichung der konkurrierenden Geschichtsschreibung im Osten und konfrontierte die DDRGeschichtswissenschaft doch gleichermaßen mit der Herausforderung einer systemsprengenden Deutungskonkurrenz. Im Osten erlag man dem selbst erzeugten Glauben an die Stabilität »einer der sozialistischen Gesellschaft gemäßen, voll ausgebildeten, produktiven Geschichtswissenschaft ${ }^{60}$, die durch ihre dosierte Integration in die internationale Fachgemeinschaft ihren Beitrag zur wachsenden Ausstrahlung des Sozialismus leisten könne, und bedrohte so mit der Kooperation zugleich die eigene Abgrenzungsidentität im Kern.

Wie das hier präsentierte Beispiel zeigt, läßt sich auf der mit den Polen Konfrontation und Kooperation markierten Skala die deutsch-deutsche Beziehungsgeschichte auf dem Feld der Historiographie nur eingeschränkt erfassen. Dieser Beurteilungsmaßstab versagt gerade dort, wo er am plausibelsten erscheint, nämlich beim Übergang von der fachlichen Abgrenzung zur Wiederannäherung, weil er die ganz unterschiedliche Funktion dieses Umschwungs in beiden Teilhistoriographien nicht angemessen zu erfassen erlaubt. Im Alltagsverständnis setzt eine Beziehung zwischen zwei Partnem logischerweise deren Selbständigkeit voraus. Im deutsch-deutschen Sonderfall aber geht es um eine Beziehung, die für die eine, östliche Seite zuallererst Verselbständigungsgeschichte bedeutete. Nur in der Abgrenzung fand sie zu

59 ABBAW, ZIG 703/9, WB DDR-Geschichte im ZIG der AdW, Bericht über das Arbeitstreffen in der Fernuniversität Hagen (BRD), 30.9.-3.10.88, 10.10.1988.

60 Walter SCHMIDT, Zur Geschichte der DDR-Geschichtswissenschaft vom Ende des zweiten Weltkriegs bis zur Gegenwart, in: Beiträge zur Geschichte der Arbeiterbewegung 27 (1985), S. 614-633. 
ihrer fachlichen Eigenidentität - und mußte sie mit der Annäherung allmählich mehr und mehr wieder einbüßen. In dieser strukturellen Differenz zwischen der politisch gebundenen ostdeutschen und der ihren eigenen Gesetzen folgenden westdeutschen Geschichtswissenschaft kommt ein Ungleichgewicht zum Ausdruck, das sich mit dem Begriff der asymmetrischen Beziehung nicht hinreichend erfassen läßt. Es zwingt vielmehr dazu, im Übergang der beiden deutschen Geschichtssysteme von der Konfrontation zur Kooperation zugleich auch den schleichenden Wechsel von der Konstituierung zur Erosion der staatssozialistischen Historiographie überhaupt zu erkennen. Es verdeutlicht zugleich, daß der Denkansatz einer asymmetrisch verflochtenen Transfer- und Beziehungsgeschichte bei aller analytischen Ergiebigkeit axiomatisch der Entspannungsphase zweier koexistierender und auf unabsehbare Dauer angelegter Systeme von Ost und West verhaftet bleibt.

Neben die Beziehungsgeschichte der beiden deutschen Geschichtswissenschaften muß daher die Geschichte ihrer Verselbständigung treten, die neben den inhaltlichen und biographischen Parallelen zweier asymmetrisch aufeinanderbezogenen Nationalhistoriographien bzw. Teilhistoriographien auch deren grundsätzliche Andersartigkeit als unterschiedlich strukturierte Wissenschaftssysteme analytisch zu erfassen vermag. 